Supporting Information

\title{
Defect Passivation in Hybrid Perovskite Solar Cells by Tailoring the Electron Density Distribution in Passivation Molecules
}

Deyu Xin a,b, Shujie Tie a,b, Ruihan Yuan ${ }^{b}$, Xiaojia Zheng b,*, Jianguo Zhu ${ }^{a,{ }^{*}}$, Wen-Hua Zhang ${ }^{\text {b,* }}$

a Department of Materials Science, Sichuan University, Chengdu 610064, China

b Sichuan Research Center of New Materials, Institute of Chemical Materials, China Academy of Engineering Physics, 596 Yinhe Road, Shuangliu, Chengdu 610200, China

\section{Corresponding Author}

* Email: xiaojia@caep.cn (X. Z.)

*Email: nic0400@scu.edu.cn (J. Z.)

* Email: whzhang@caep.cn (W.-H. Z.) 

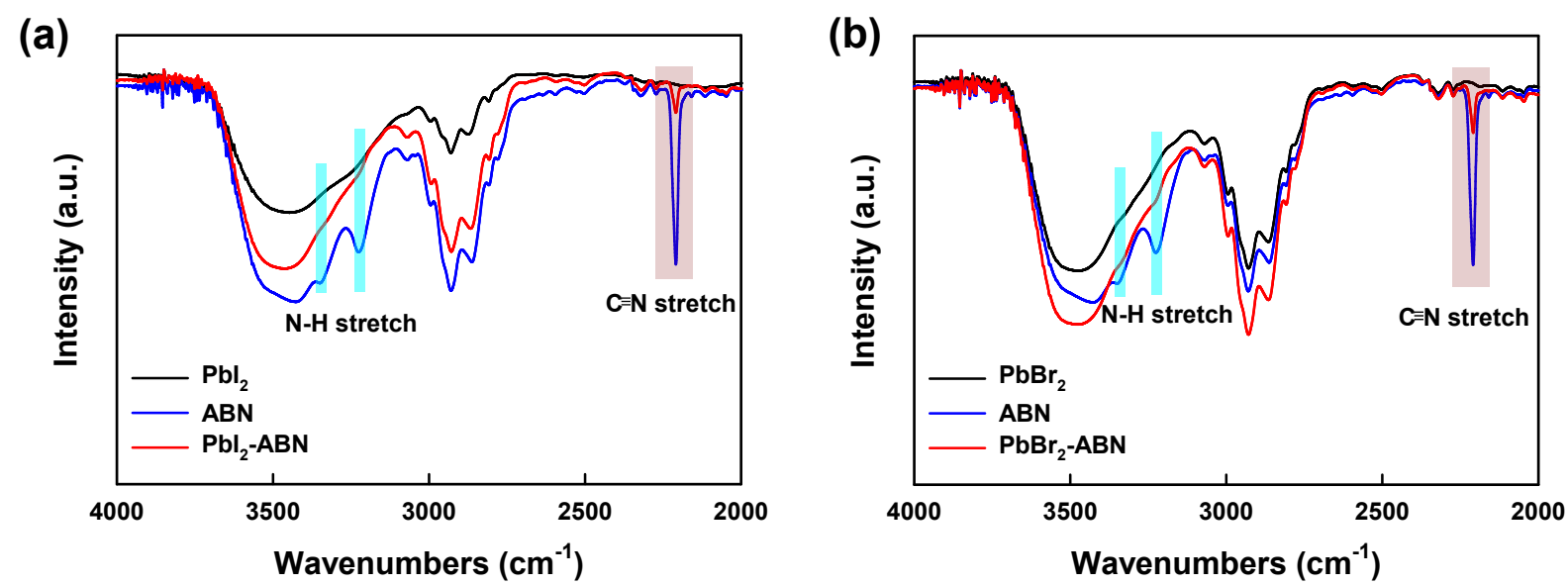

Figure S1. FTIR spectra of (a) $\mathrm{PbI}_{2}, \mathrm{ABN}$ and $\mathrm{PbI}_{2}-\mathrm{ABN}$ blend and (b) $\mathrm{PbBr}_{2}, \mathrm{ABN}$ and $\mathrm{PbBr}_{2}-\mathrm{ABN}$ blend. 


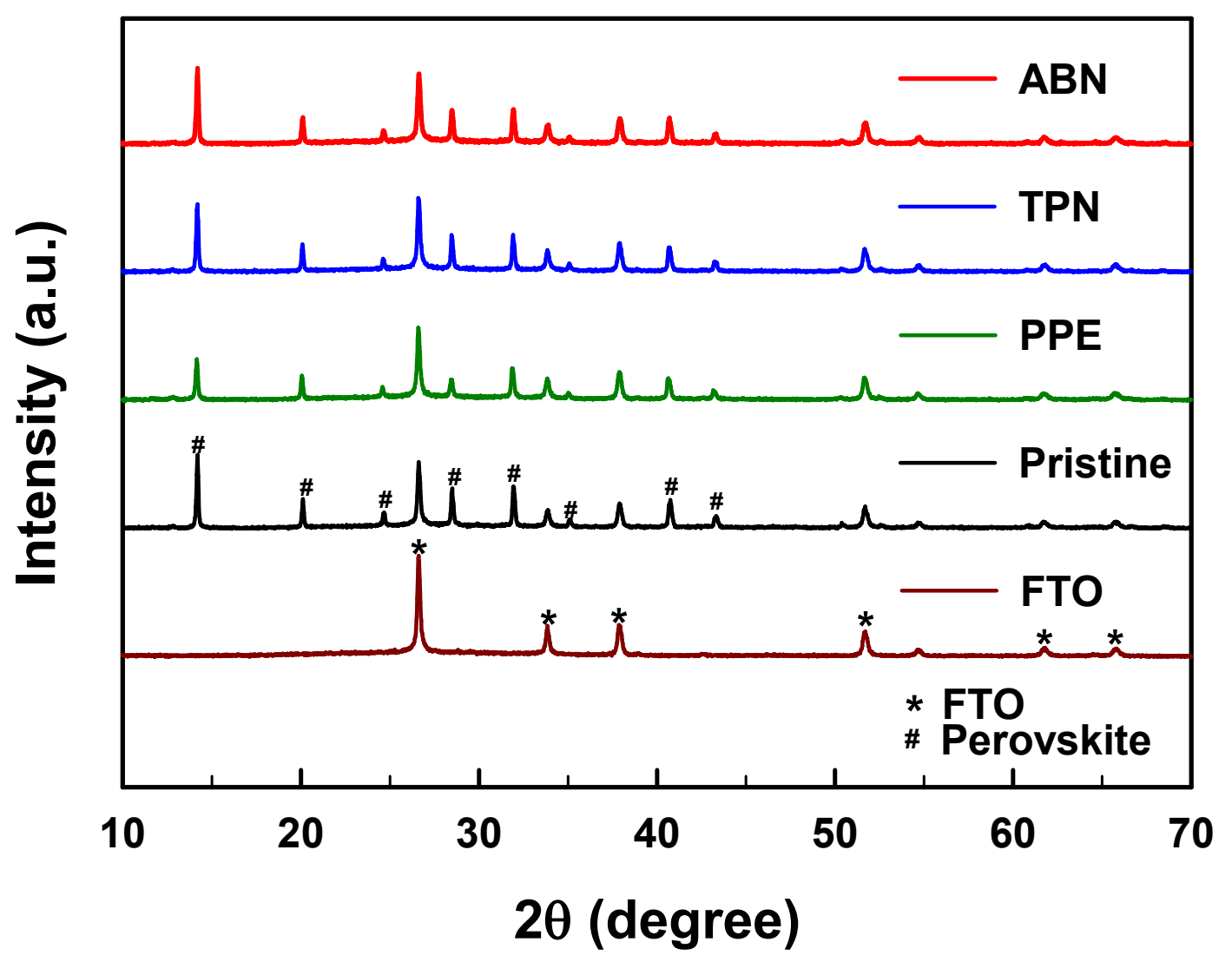

Figure S2. XRD patterns of perovskite films with and without the passivation molecules. 

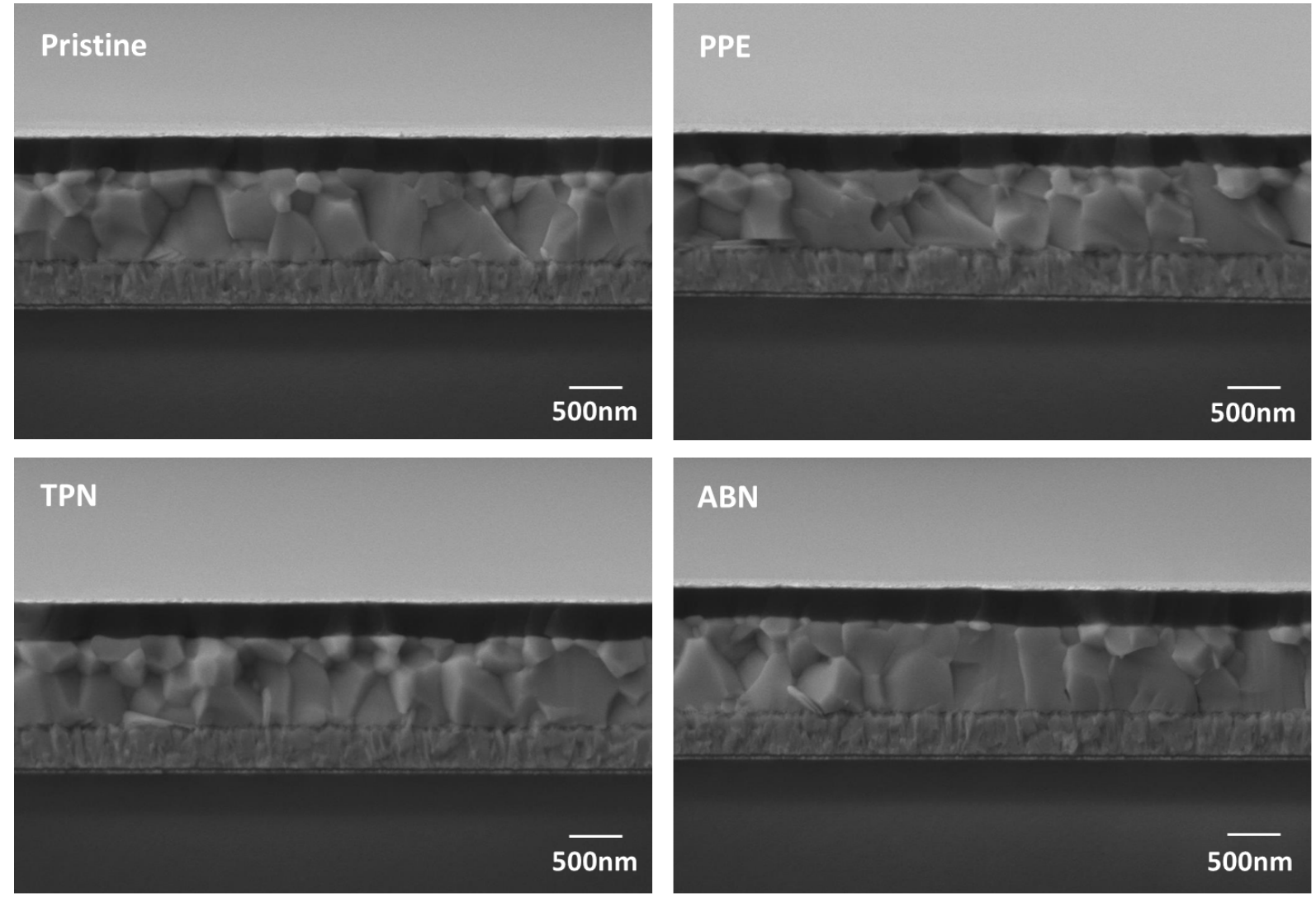

Figure S3. Cross-section SEM images of PSCs. 

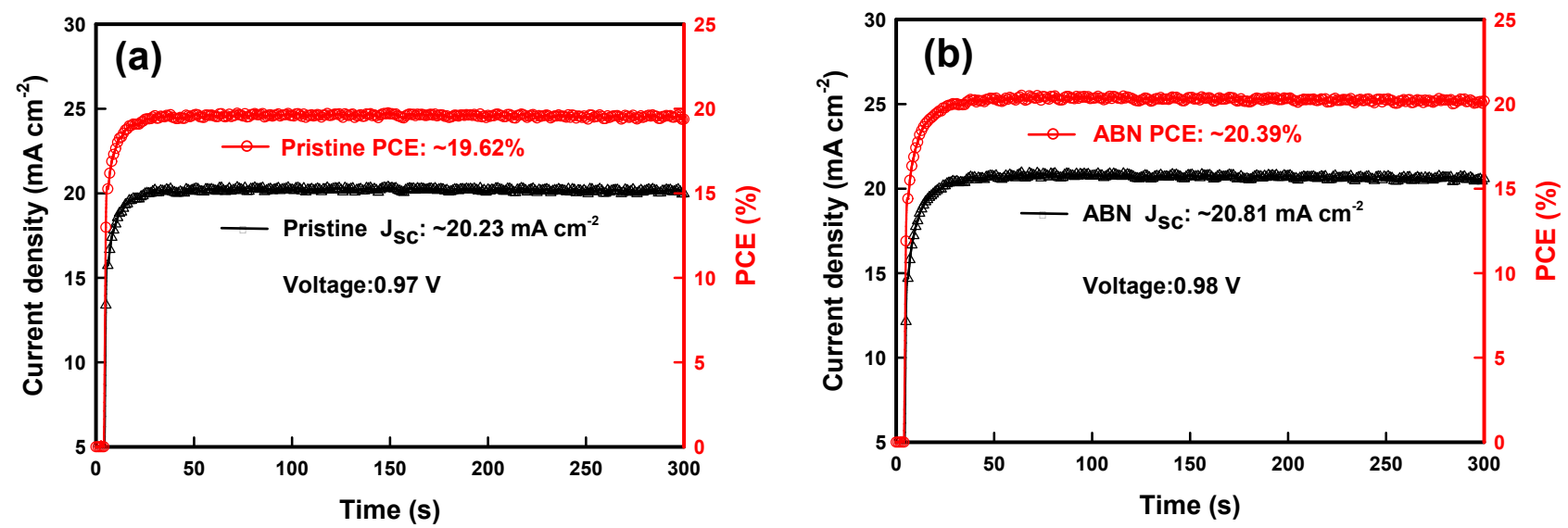

Figure S4. The stabilized output of the efficiency (red) and current density (black) as a function of time at the maximum power point of (a) Pristine and (b) CsFAMA-ABN device under simulated 1 sun illumination. 

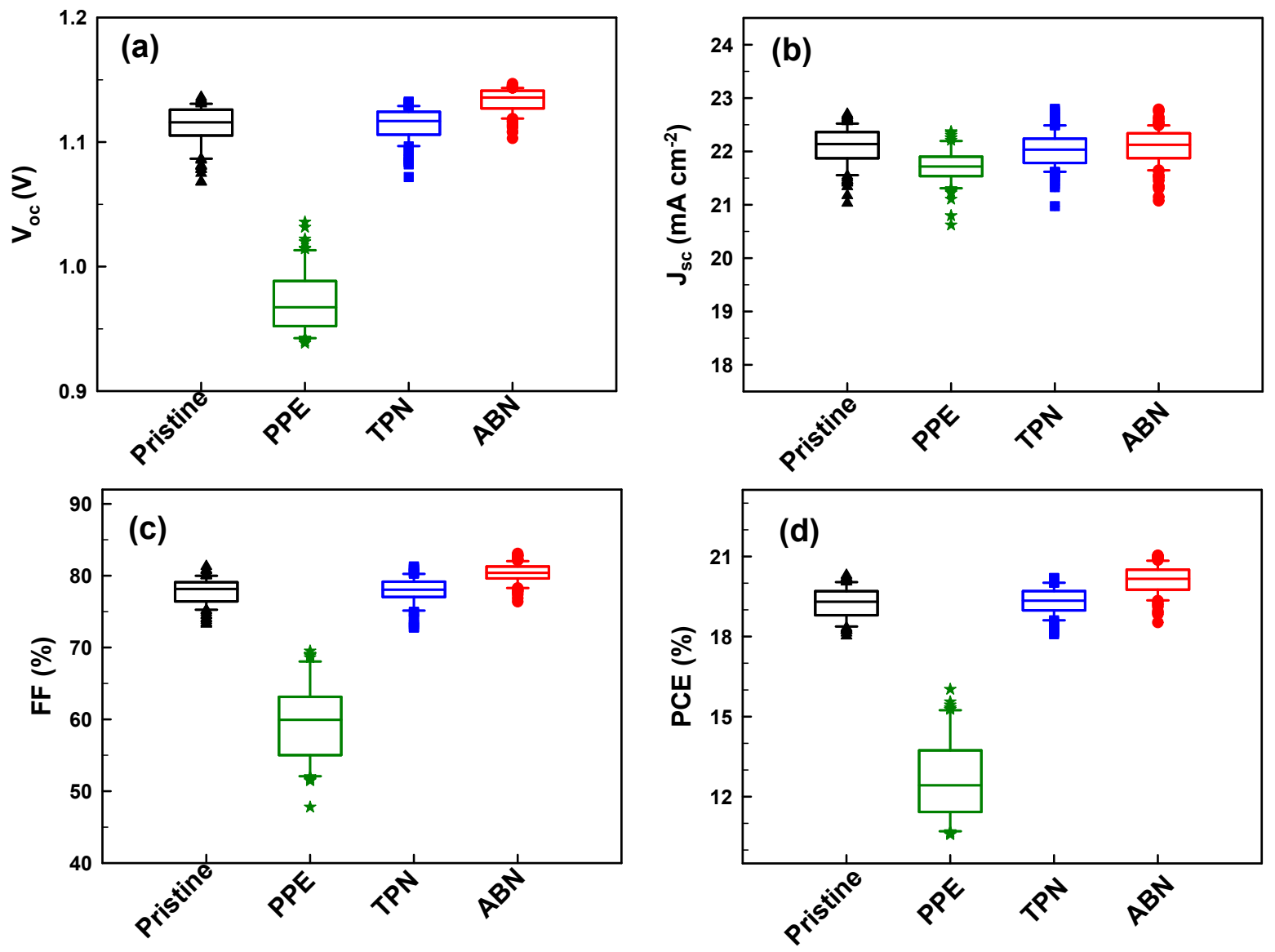

Figure S5. Performance distribution of the solar cells with and without the passivation molecules: (a) $\mathrm{V}_{\mathrm{oc}}$, (b) $\mathrm{J}_{\mathrm{sc}}$, (c) fill factor, and (d) efficiency. 


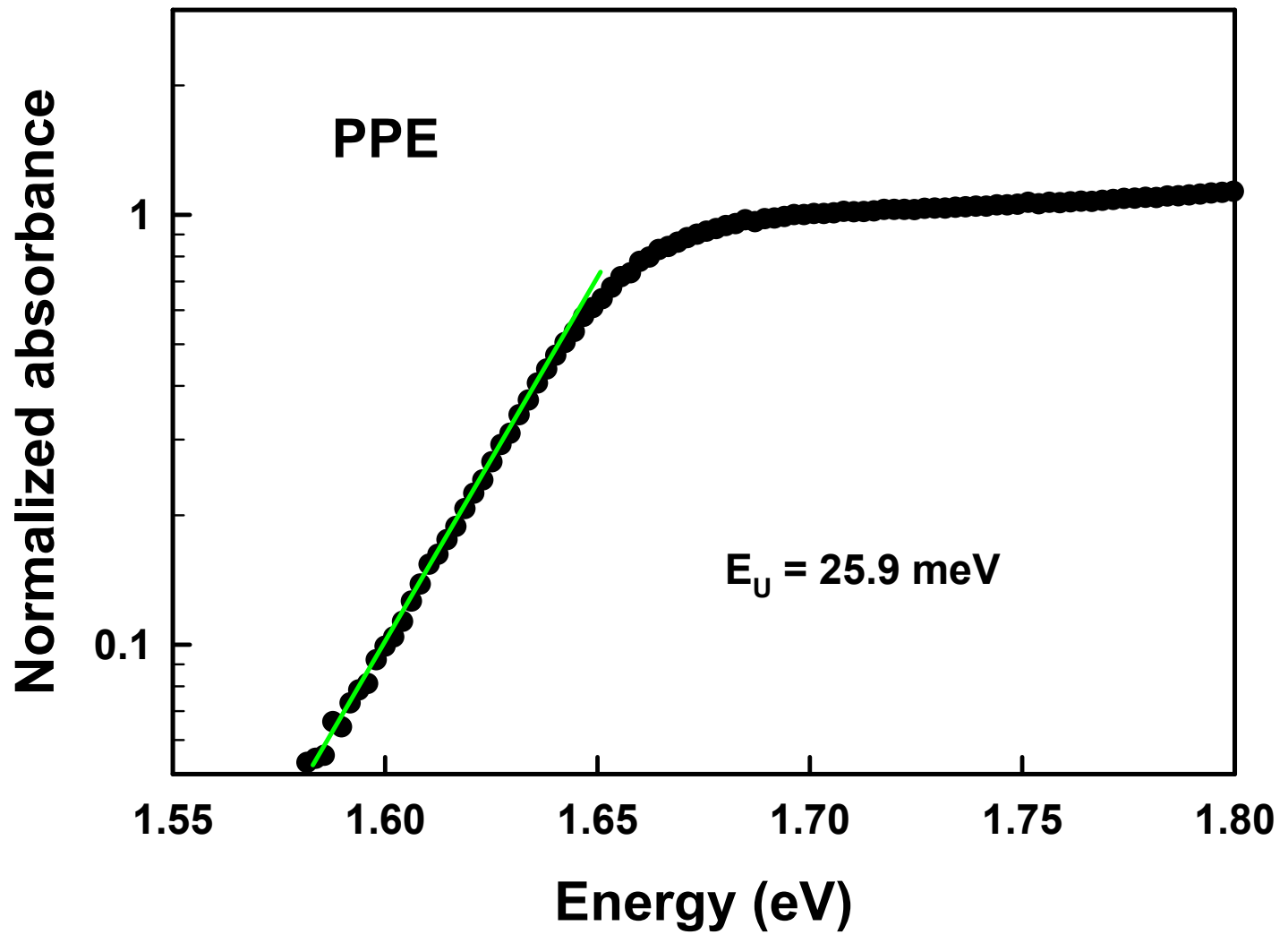

Figure S6. Normalized absorbance with the linear fit (green line) of the Urbach tail for CsFAMAPPE. 


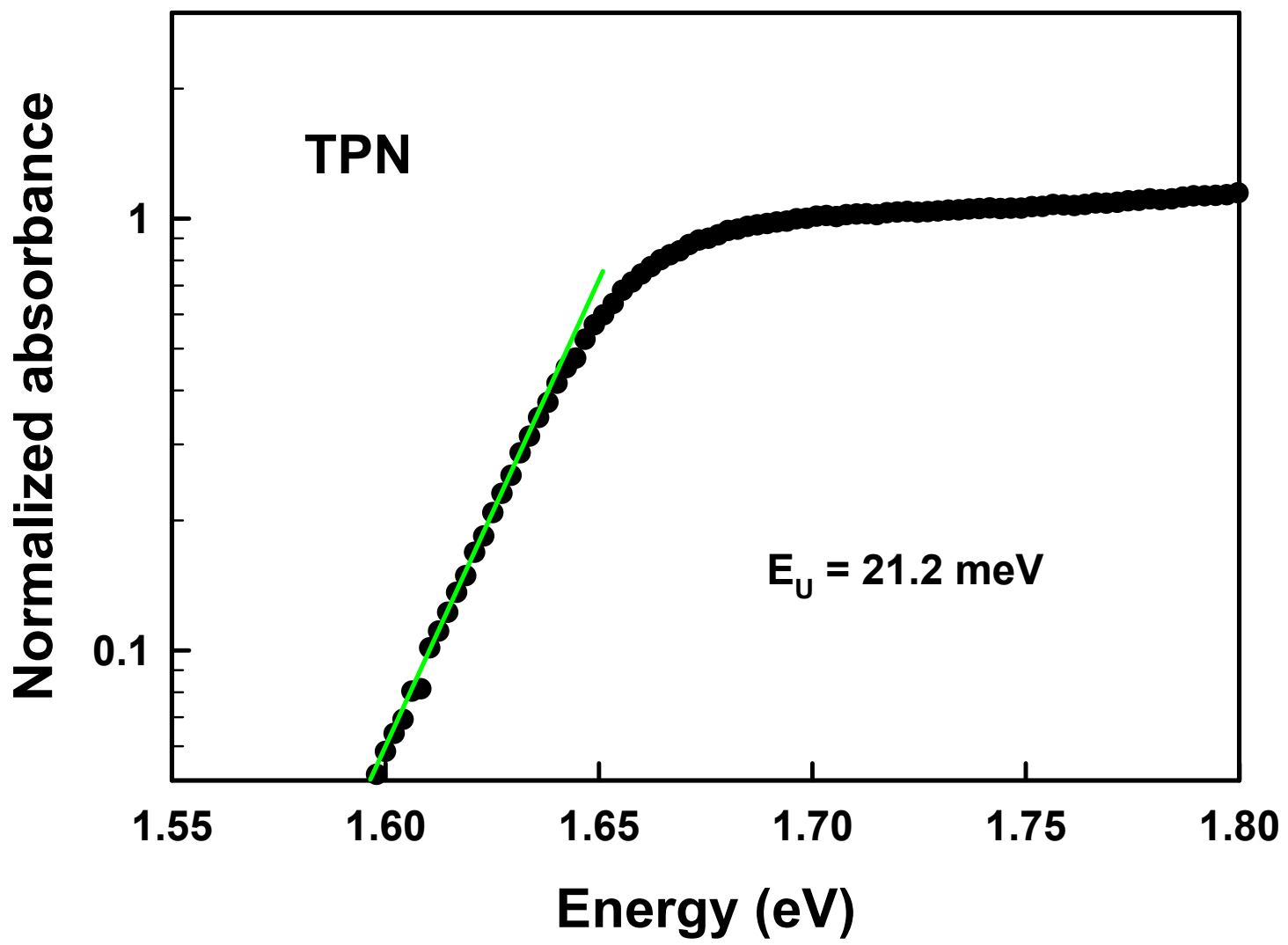

Figure S7. Normalized absorbance with the linear fit (green line) of the Urbach tail for CsFAMATPN. 


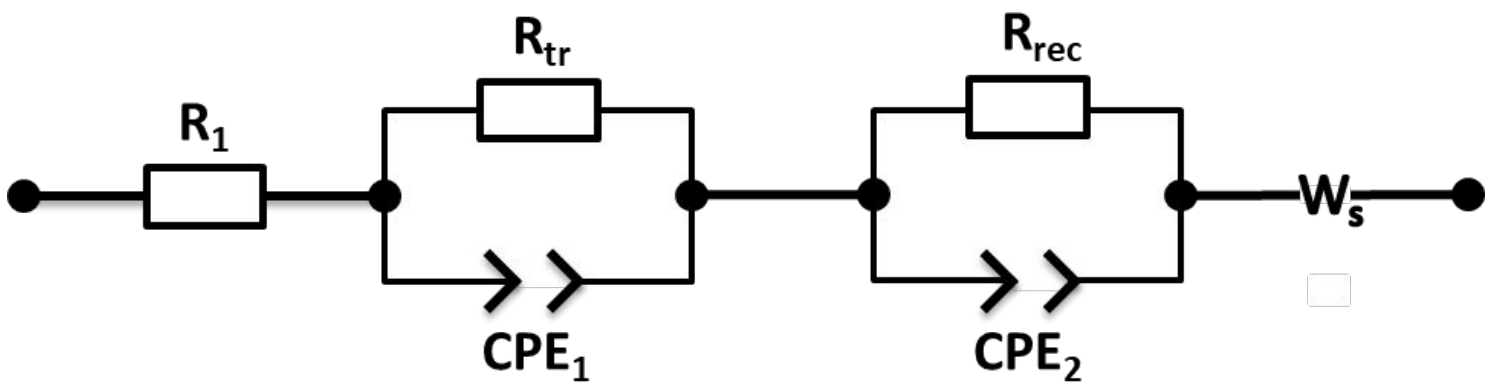

Figure S8. Equivalent circuit diagram used to fit the Nyquist plots. 


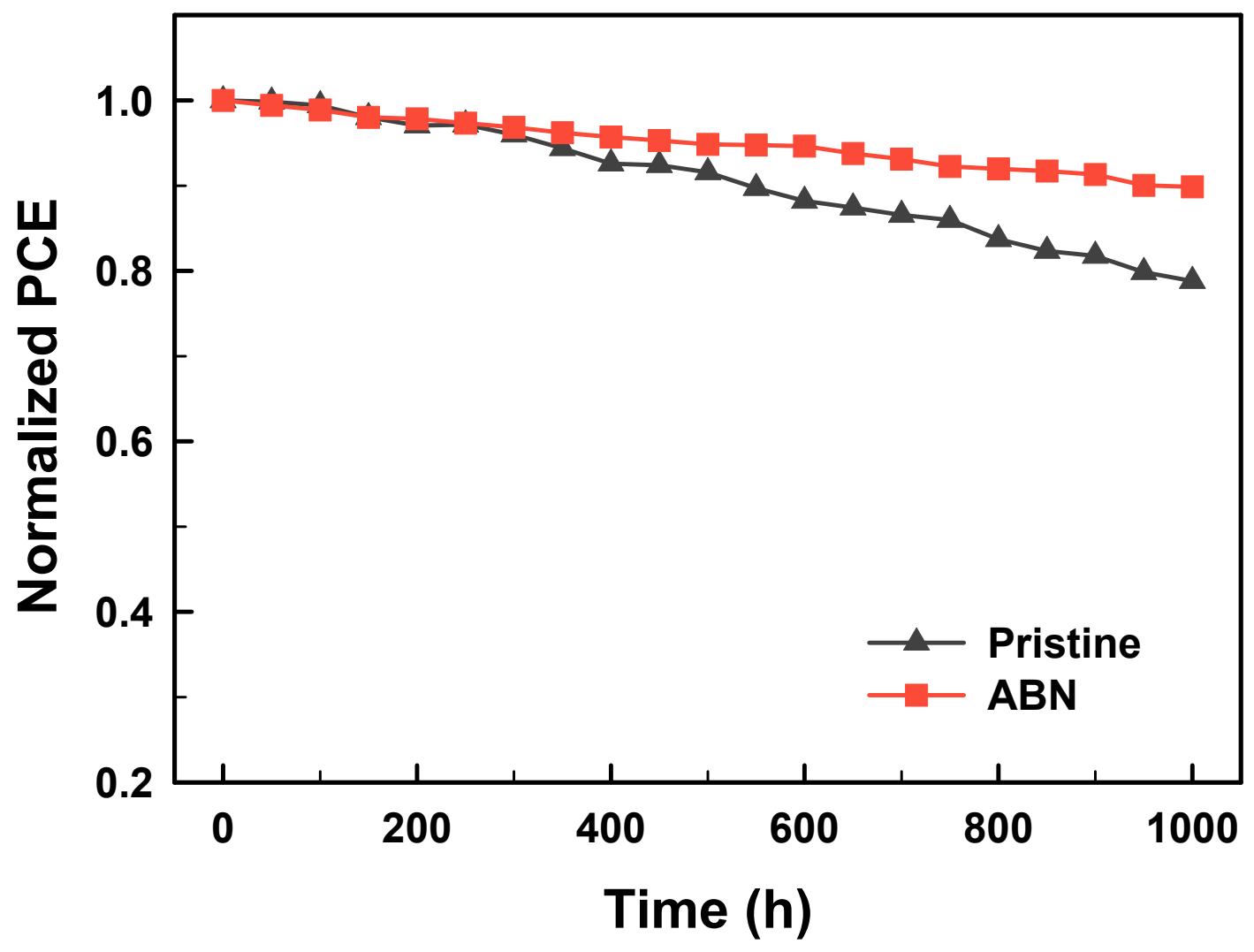

Figure S9. Shelf stability of PSCs stored in air under a $\mathrm{RH} \sim 50 \%$ at $23{ }^{\circ} \mathrm{C}$ without encapsulation. 
Table S1. Parameters of the time-resolved photoluminescence (TRPL) spectroscopy for perovskite films.

\begin{tabular}{|l|l|l|l|l|l|}
\hline Samples & $\tau_{\text {ave }}(\mathrm{ns})$ & $\tau_{1}(\mathrm{~ns})$ & Amplitude & & Amplitude \\
of $\tau_{1}(\%)$ & $\tau_{2}(\mathrm{~ns})$ & of $\tau_{2}(\%)$ \\
\hline Pristine & 1481.2 & 222.6 & 29.5 & 2007.8 & 70.5 \\
\hline PPE & 41.6 & 12.4 & 52.9 & 74.3 & 47.1 \\
\hline TPN & 1503.2 & 140.1 & 27.3 & 2015.1 & 72.7 \\
\hline ABN & 1755.4 & 209.6 & 23.2 & 2222.3 & 76.8 \\
\hline
\end{tabular}

